\section{Templating Effect of Lipid Membranes on Alzheimer's Amyloid Beta Peptide}

\author{
Canay Ege, ${ }^{[a]}$ Jaroslaw Majewski, ${ }^{[b]}$ Guohui Wu, ${ }^{\left[{ }^{[a]}\right.}$ \\ Kristian Kjaer, ${ }^{[c]}$ and Ka Yee C. Lee ${ }^{*[a]}$
}

Alzheimer's disease is characterized by amorphous deposits in the brain, with the major component being the 40- to 43-residue amyloid beta $(A \beta)$ peptide. ${ }^{[1,2]}$ In its oligomeric form, $A \beta$ is believed to be a causal agent for Alzheimer's disease. ${ }^{[3]}$ While incubation studies require millimoles of $A \beta$ for aggregate formation, only micromolar concentrations are needed in the presence of lipid vesicles, ${ }^{[4-6]}$ suggesting a possible role of lipids in $A \beta$ aggregation. This has led us to examine the interaction between $A \beta 40$ and three lipids with identical tails, but different head groups: zwitterionic dipalmitoyl phosphatidyl choline (DPPC), anionic dipalmitoyl phosphatidyl glycerol (DPPG), and cationic dipalmitoyl trimethyl ammonium propane (DPTAP). Although cationic lipids are absent in cell membranes, the use of DPTAP is critical to understanding the role of charge on peptide association. To mimic the outer leaflet of a membrane, monolayers at the air-water interface were held at a (bilayer equivalent ${ }^{[7]}$ ) pressure of $30 \mathrm{mN} \mathrm{m}^{-1} \cdot{ }^{[8]}$ Our previous isotherm data show that $A \beta$ inserts into all three lipids at surface pressures below $25 \mathrm{mN} \mathrm{m}^{-1}$ at $30^{\circ} \mathrm{C}^{[9]}$ At $30 \mathrm{mN} \mathrm{m}^{-1}$, there is insertion for DPPG and DPTAP, but not for DPPC. Fluorescence microscopy (FM) measurements show that $A \beta$ insertion disrupts condensed domains, increasing the area fraction of the disordered phase. However, FM and isotherm measurements do not provide molecular-scale details. Here, we report the first X-ray reflectivity $(X R)$ and grazing incidence $X$-ray diffraction (GIXD) on lipid/A $\beta$ systems. Not only do these data pinpoint the location of $A \beta$ in the lipid matrix, they also unveil an intriguing templating effect of DPPG on $A \beta$.

We first examined $A \beta$ at the bare air-water interface. From XR (Figure 1), we obtain a surface layer $18.6 \pm 1.6 \AA$ thick with normalized electron density (ED), $\rho_{A \beta} / \rho_{\text {water }}=1.26$ (see inset). Here, $\rho_{A \beta}$ is the average A $\beta$ ED, and $\rho_{\text {water }}$ is that of the subphase. The roughness of the air-peptide interface is $2.8 \AA$, explicable by capillary waves, while that of the peptide-water interface is $9.1 \AA$, indicating a diffuse ED profile extending into the subphase. $A \beta$ also exhibits lateral ordering, giving rise to Bragg peaks ${ }^{[10]}$ at $Q_{x y}=1.32 \AA^{-1}$ and $0.17 \AA^{-1}$ (Figure $1 \mathrm{~b}$ ), with

[a] Dr. C. Ege, G. Wu, Prof. K. Y. C. Lee

Department of Chemistry, The James Franck Institute and

The Institute for Biophysical Dynamics, The University of Chicago Chicago IL 60637 (USA)

Fax: $(+1) 773-702-0805$

E-mail: kayeelee@uchicago.edu

[b] Dr. J. Majewski

Los Alamos Neutron Scattering Center, Los Alamos National Laboratory Los Alamos, NM 87545 (USA)

[c] Dr. K. Kjaer

Materials Research Department, Risø National Laboratory Roskilde (Denmark)
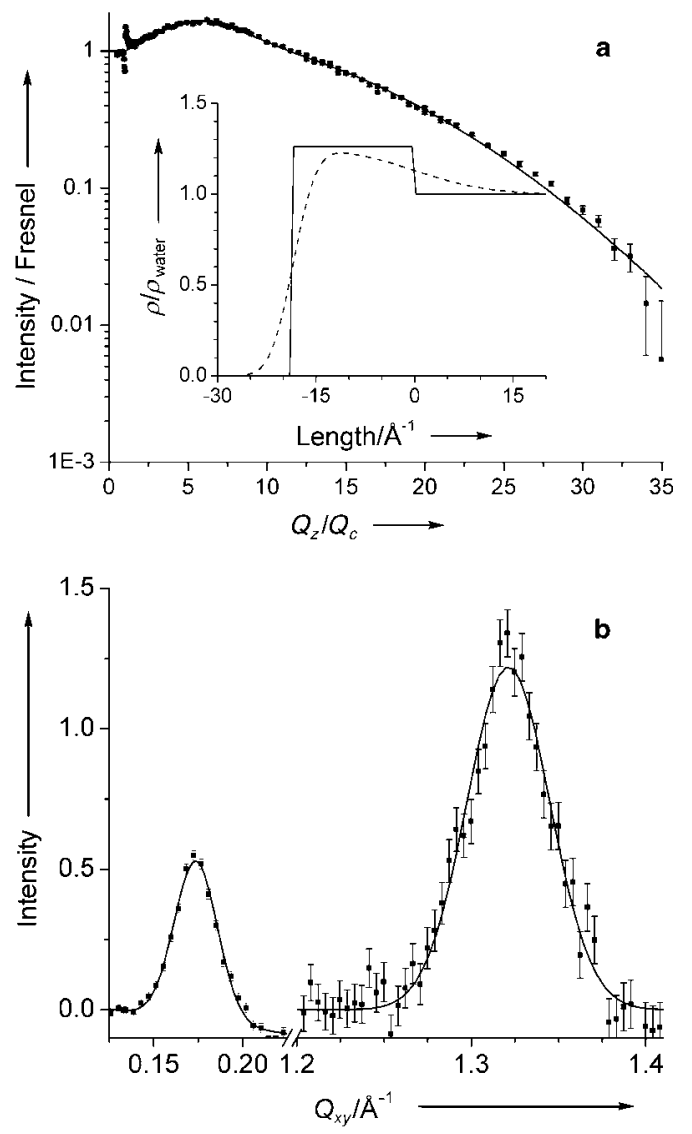

Figure 1. $X$-ray scattering on pure $A \beta 40$ at the air-water interface at a surface pressure of $13 \mathrm{mN} \mathrm{m}-1$ and $30^{\circ} \mathrm{C}$; a) XR data and fit. The inset shows the corre sponding normalized ED models in smeared (by interfacial roughness) and unsmeared forms. $Q_{z}$ is the momentum transfer vector and $Q_{c}$ is the momentum transfer vector corresponding to the critical angle for total external reflection from the water subphase; b) GIXD data and fit showing the two Bragg peaks obtained, indicating spontaneous in-plane ordering of pure peptide at the airliquid interface. $\mathrm{Q}_{\mathrm{xy}}$ is the in-plane momentum transfer vector.

$d$-spacings of $4.76 \AA$ and $36.2 \AA$, and in-plane coherence lengths (signifying the extent of in-plane order) of $100 \AA$ and $210 \AA$, respectively. The ratio of the Bragg peak integrated intensities, $I_{1.32} / I_{0.17}$, is $\cong 4$. Analysis of the Bragg $\operatorname{rod}^{[11]}$ for the peak at $Q_{x y}=1.32 \AA^{-1}$ indicates an out-of-plane thickness of $13 \pm 5 \AA$ for the $A \beta$ scatterer. Although we do not expect fibril formation at $250 \mathrm{nM}^{[12]}$ in our measurements, the $d$-spacing of $4.76 \AA$ is identical to the $\beta$-sheet spacing between $A \beta$ monomers of an amyloid fibril. ${ }^{[13,14]}$ This suggests that $A \beta$ adopts $a$ $\beta$-sheet conformation and is likely to be parallel to the interface. The $36.2 \AA$ spacing is probably due to a supramolecular structure.

$A \beta$ adsorbs to and orders at the bare water surface. How, then, would a lipid monolayer affect $A \beta$ surface adsorption and ordering? XR measurements were taken before and after $A \beta$ injection with the monolayer held at $30 \mathrm{mN} \mathrm{m}^{-1}$ (Figure 2). DPTAP and DPPG monolayers can be modeled using two slabs, ${ }^{[15]}$ one for the ED of the tails, $\rho_{\mathrm{t}}\left(\rho_{\mathrm{t}} / \rho_{\text {water }} \cong 0.95\right.$ in Figures $2 \mathrm{a}^{\prime}$ and $\left.2 \mathrm{~b}^{\prime}\right)$ and the other for the head group, $\rho_{\mathrm{h}}\left(\rho_{\mathrm{h}} /\right.$ $\rho_{\text {water }} \cong 1.3$ for DPTAP and $\cong 1.4$ for DPPG). Upon A $\beta$ injection, 

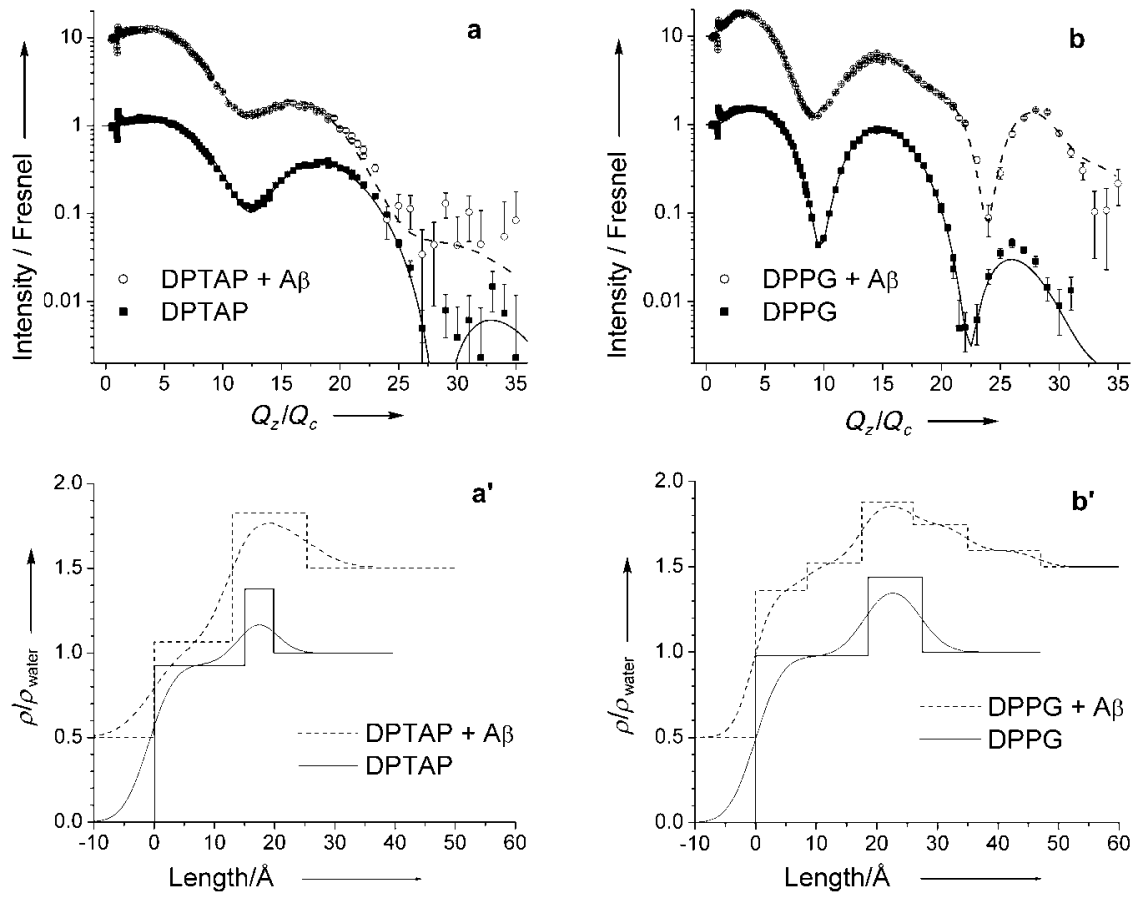

Figure 2. $X R$ data and fit, and the corresponding ED distribution perpendicular to the interface for DPTAP $\left(a, a^{\prime}\right)$ and $\operatorname{DPPG}\left(b, b^{\prime}\right)$ at $30 \mathrm{mNm} \mathrm{m}^{-1}$ and $30^{\circ} \mathrm{C}$ with (O) and without (a) $A \beta 40$. The solid and dashed lines in (a) and (b) are fits to the XR data. The corresponding normalized ED models are shown in smeared (by interfacial roughness) and unsmeared forms in $\left(a^{\prime}\right)$ and $\left(b^{\prime}\right)$. The reflectivities and profiles have been offset for clarity. chains ${ }^{[18,19]}$ with $a=4.83 \AA$, and $A=20.2 \AA^{2}$. When $A \beta$ is injected, the unit cell becomes distorted, as the single peak splits into two. The expanded unit cell has dimensions $\quad a=4.94 \AA, \quad \gamma=$ $118.7^{\circ}$ and $A=21.4 \AA^{2}$. For DPPG, the peptide preferentially inserts into the disordered phase and disrupts the condensed phase ordering at the domain boundary (Figure $4 \mathrm{~b}$ ). The observed expansion of the DPPG unit cell reflects a lowering of lipid ordering. In addition, $A \beta$ gives rise to an extra peak at $Q_{x y}=1.32 \AA^{-1}$, the same location as one of the two peptide peaks, but with an intensity 2.5 times larger, and a coherence length ( $480 \AA)$ approximately five times larger. DPPG thus enhances $A \beta$ ordering along the shorter $d$-spacing direction. Curiously, this addition- the XR profile for DPTAP can still be fit by two slabs, but the ED of the second slab is lower, the first slab thicker, and the roughness greater than in pure DPTAP (Figure $2 a^{\prime}$ ). Here, the first slab encompasses the tail/peptide, head/peptide, and the peptide portion protruding into the subphase ${ }^{[16]}$ while the second corresponds to a portion of the tail region. 3- and 4slab models have been used but do not yield better fits. Unlike DPTAP, the profile for mixed DPPG/A $\beta$ is more complicated and can only be fitted by a 5-slab model (see Figure $2 \mathrm{~b}^{\prime}$ ); similar results have been obtained by an independent free-form Bspline method. ${ }^{[17]}$ 2- to 4 -slab models were also exhaustively tested, but did not yield satisfactory fits. The lowered $\rho_{\mathrm{t}}$ values ( 0.58 for DPTAP, 0.80 for DPPG) in the mixed film suggest that $\mathrm{A} \beta$ is partially inserted, and agree quantitatively with the $35 \%$ and $14 \%$ area increases found in our insertion assays, ${ }^{[9]}$ respectively. For zwitterionic DPPC, there is no detectable change in the $X R$ profile upon $A \beta$ injection.

GIXD shows that $A \beta$ affects lipid ordering. Pure DPTAP shows two Bragg peaks (Figure $3 \mathrm{a}$ ), indicating a distorted hexagonal cell formed by hydrocarbon tails with dimension $a=$ $5.06 \AA$, area $A=22.4 \AA^{2}$, and $\gamma=118.9^{\circ}$, where $\gamma$ is the angle between the base vectors of the unit cell. Upon $A \beta$ injection, the peaks are shifted to higher $Q_{x y}$ as the unit cell contracts to $a=4.98 \AA, \gamma=117.9^{\circ}$ and $A=21.9 \AA^{2}$ (Figure $3 \mathrm{a}$, open circles). We know from $F M$ data ${ }^{[9]}$ that $A \beta$ adsorbs to the condensed phase of DPTAP. The contraction can therefore be understood in terms of a multivalent ion condensation effect. For pure DPPG (Figure $3 \mathrm{~b}$ ), we observe a single Bragg peak at $Q_{x y}=$ $1.50 \AA^{-1}$, corresponding to a hexagonal arrangement of alkyl
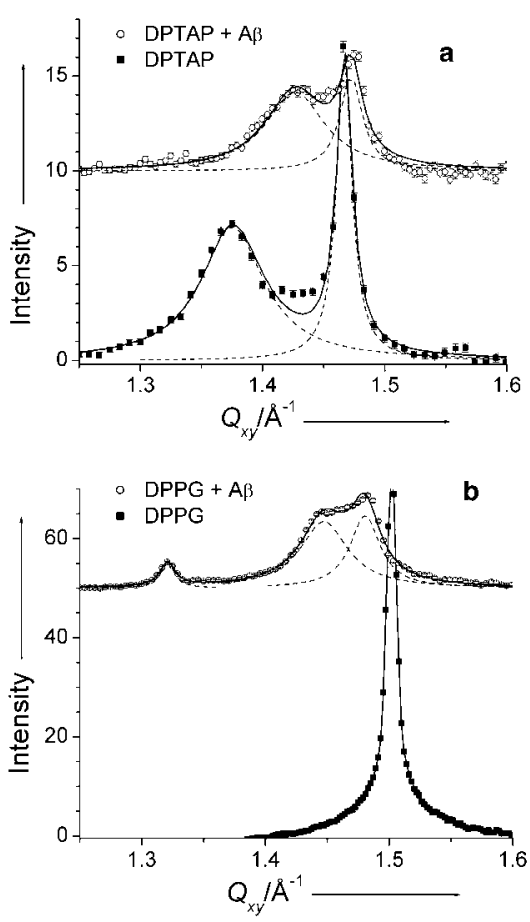

Figure 3. Background-subtracted GIXD spectra on water at $30^{\circ} \mathrm{C}$ and $30 \mathrm{mN} \mathrm{m} \mathrm{m}^{-1}$ for a) DPTAP, and b) DPPG with (O) or without (घ) A $\beta$. For clarity, the data have been offset vertically. The third peak around $Q_{x y}=1.32 \AA^{-1}$ in (b) is due to $A \beta$. Due to different molecular geometries, DPTAP exhibits a distorted hexagonal unit cell and DPPG a hexagonal unit cell at the same surface pressure. Injection of $A \beta$ results in a contraction of the unit cell for DPTAP (peaks are closer together and shifted to the right) but an expansion for DPPG (the single peak is split into two at lower $\mathrm{Q}_{\mathrm{xy}}$ ). Moreover, only for DPPG (b), an additional peak at $\mathrm{Q}_{\mathrm{xy}}=1.32 \AA^{-1}$, corresponding to one of the pure peptide peaks is, observed. 

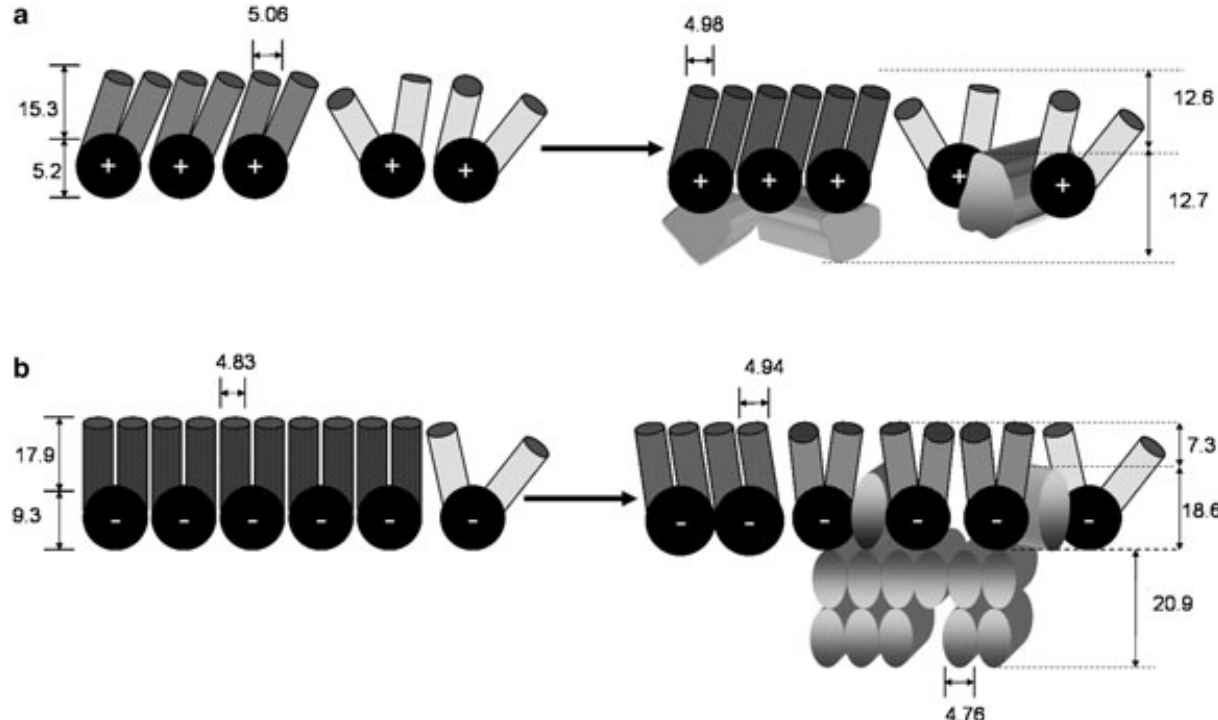

Figure 4. a) DPTAP and b) DPPG monolayers before (left hand side) and after (right hand side) the injection of $A \beta 40$ into the subphase. All lengths are in $\AA$. The lipid tail groups are shaded from dark (ordered) to light (disordered). For both DPPG and DPTAP, $A \beta$ reduces the area fraction of the condensed phase lipids, as illustrated by the conversion of lipid molecules from being in an ordered phase to a disordered one. a) The tilt of the tail group in the condensed phase of DPTAP decreases as the peptide layer beneath it (FM data ${ }^{[9]}$ ) acts as a multivalent ion, tightening the unit cell. b) Unlike in the DPTAP case, FM data indicate that there is no peptide beneath the condensed phase region of $D P P G$, thus the condensing effect observed for DPTAP is not present here. Instead, the tilt of the tail group in the condensed phase of DPPG increases due to the disordering effect of the peptide. For DPPG, the adsorbed A $\beta$ is ordered and gives rise to a d-spacing of $4.76 \AA$, identical to the $\beta$-sheet spacing between peptide monomers in amyloid fibrils. Therefore, it is likely that $A \beta$, adsorbed to a DPPG monolayer, is in a $\beta$-sheet conformation with the sheets aligning parallel to the interface. A similar $\beta$-sheet conformation for $A \beta$ adsorbed to a monolayer has been detected with infrared spectroscopy. ${ }^{[22]}$

al peak is only observed for DPPG, and the peptide peak at $Q_{x y}=0.17 \AA^{-1}$ is not detected in any of the cases.

The fact that $A \beta$ inserts into both DPPG and DPTAP but exhibits $2 \mathrm{D}$ peptide ordering only in DPPG indicates that the conformation of $A \beta$ in the presence of DPPG must be similar to that of pure $A \beta$. The lack of any peptide peak in DPTAP, on the other hand, suggests a different peptide conformation and/or orientation in the presence of an opposite head group charge. Thus, the electrostatic environment greatly influences peptide association, which in turn affects the out-of-plane thickness of the associated peptide layer. XR shows that for DPPG this thickness is $39.7 \AA$, but only $12.7 \AA$ for DPTAP. While $12.7 \AA$ agrees well with a single layer of $A \beta$ associated with DPTAP (Figure $4 \mathrm{a}$ ), the $39.7 \AA$ thickness indicates that apart from insertion, there must also be peptide adsorption underneath the DPPG head group. The adsorbed population is likely made up of two layers (Figure $4 \mathrm{~b}$ ), and gives rise to the peptide peak observed in DPPG.

It is apparent that anionic DPPG enhances $A \beta$ ordering, while DPPC and DPTAP inhibit it. One scenario for $A \beta$ aggregation is that anionic lipids, normally at the inner leaflet, may get exposed to the outer leaflet due to membrane damages. Their presence can help partition $A \beta$ close to the membrane surface and fasten oligomerization by reducing the peptide monomer's search for one another from a three- to a two-dimensional one.

\section{Experimental Section}

DPPC, DPPG and DPTAP were obtained from Avanti Polar Lipids (Alabaster, AL) in powder form and dissolved in chloroform/ methanol mixtures to prepare spreading solutions. ${ }^{[9]}$ Measurements were taken on a temperature-controlled film balance with a pure water (Milli-Q, Millipore, Bedford, MA) subphase at $30^{\circ} \mathrm{C}$ and a measured $\mathrm{pH}$ of 5.5. Under these conditions, $A \beta$ (with six positive and six negative charges) has no net charge. $A \beta$ was purchased from Anaspec, Inc. (San Jose, CA) and dissolved in dimethyl sulfoxide at least two hours prior to experiments at $5 \mathrm{mg} \mathrm{mL}^{-1}$ to ensure that aggregates were broken up $^{[20]}$ as confirmed by size exclusion chromatography. The final concentration of $A \beta$ in the subphase was $250 \mathrm{~nm}^{[12]}$ Details of the $A \beta$ injection protocol ${ }^{[9]}$ and the X-ray scattering apparatus ${ }^{[18,21]}$ are given elsewhere.

\section{Acknowledgements}

We acknowledge $B W 1$ beamtime at HASYLAB, DESY, Germany and financial support under the DanSync programme of the Danish Natural Science Research Council. C.E. was supported by Alzheimer's Association (IIRG-9901175), American Health Assistance Foundation (A-1999057) and the University of Chicago MRSEC program of the NSF (DMR0213745). J. M. was supported (in part) by Los Alamos National Laboratory under DOE contract W7405-ENG-36, and by the DOE Office of Basic Energy Sciences. G.W. acknowledges the support of Burroughs Wellcome Fund Interfaces No: 1001774. K.Y.C.L. is grateful for support from the Packard Foundation (99-1465), and the Sloan Foundation (BR-4028). The experimental apparatus was made possible by an NSF CRIF Grant (CHE-9816513) and DanSync.

Keywords: Alzheimer's disease - monolayers · phospholipids $\mathrm{X}$-ray diffraction $\cdot \mathrm{X}$-ray reflectivity

[1] J. Hardy, Trends Neurosci. 1997, 20, 154.

[2] D. J. Selkoe, Annu. Rev. Cell Biol. 1994, 10, 373.

[3] J. Naslund, V. Haroutunian, R. Mohs, K. L. Davis, P. Davies, P. Greengard, J. D. Buxbaum, JAMA J. Am. Med. Assoc. 2000, 283, 1571; M. Buccianti, E. Giannoni, F. Chiti, F. Baroni, L. Formigli, J. Zurdo, N. Taddei, G. Ramponi, C. M. Dobson, M. Stefani, Nature 2002, 416, 507.

[4] E. Terzi, G. Hölzemann, J. Seelig, Biochemistry 1997, 36, 14845.

[5] J. McLaurin, A. Chakrabartty, Eur. J. Biochem. 1997, 245, 355.

[6] M. Bokvist, F. Lindstrom, A. Watts, G. Grobner, J Mol Biol 2004, 335, 1039.

[7] A. Seelig, Biochim. Biophys. Acta 1987, 899, 196. 
[8] A monolayer is a good mimic since $A \beta$ inserts only partially into the membrane.

[9] C. Ege, K. Y. C. Lee, Biophys. J. 2004, 87, 1732

[10] T. Jensen, K. Kjaer in Studies in Interface Science, Vol. 11: Novel methods to study interfacial layers (Eds.: D. Möbius, R. Miller) Elsevier, Amsterdam, New York, 2001, pp. 205-254.

[11] J. Als-Nielsen, K. Kjaer in Phase Transitions in Soft Condensed Matter, Vol. B211: Reflectivity and Diffraction Studies of Liquid Surfaces and Surfactant Monolayers, NATO Advanced Study Institutes, Plenum Publishing Corp. New York, 1989, p. 113.

[12] The $A \beta$ concentration used is much lower than the critical micelle concentration $(12.5 \mu \mathrm{M})$ and the concentration necessary for fibril formation. Aggregation studies require at least two orders of magnitude higher concentration with an incubation period of days.

[13] S. B. Malinchik, H. Inouye, K. E. Szumowski, D. A. Kirschner, Biophys. J. 1998, 74, 537.

[14] T. L. Benzinger, D. M. Gregory, T. S. Burkoth, H. Miller-Auer, D. G. Lynn, R. E. Botto, S. C. Meredith, Biochemistry 2000, 39, 3491.

[15] XR data were fit using a coherent superposition of reflectivities, because when transferred onto a solid substrate and analyzed via atomic force microscopy, we see nanoscale phase separation (not picked up by FM). Additional justification is the presence of the sharp minima in Figure $2 b$.

[16] The similarity of EDs for A $\beta 40$ and the DPTAP head group justifies the use of a single slab.

[17] J. S. Pedersen, I. W. Hamley, J. Appl. Crystallogr. 1994, 27, 36.

[18] K. Kjaer, J. Als-Nielsen, C. A. Helm, L. A. Laxhuber, H. Möhwald, Phys. Rev. Lett. 1987, 58, 2224.

[19] C. Böhm, H. Möhwald, L. Leiserowitz, J. Als-Nielsen, K. Kjaer, Biophys. J. 1993, 64, 553.

[20] C. L. Shen, R. M. Murphy, Biophys. J. 1995, 69, 640.

[21] J. Majewski, R. Popovitz-Biro, W. G. Bouwman, K. Kjaer, J. Als-Nielsen, M. Lahav, L. Leiserowitz, Chem. Eur. J. 1995, 1, 304.

[22] E. Maltseva, G. Brezesinski, ChemPhysChem, 2004, 5, 1185.

Received: September 27, 2004 